\title{
Retrospective Cost Adaptive Control for Systems with Unknown Nonminimum-Phase Zeros
}

\author{
Anthony M. D’Amato, E. Dogan Sumer, and Dennis S. Bernstein*
}

\begin{abstract}
We develop a multi-input, multi-output direct adaptive controller for discrete-time, possibly nonminimum-phase, systems with unknown nonminimum-phase zeros. The adaptive controller requires limited modeling information about the system, specifically, Markov parameters from the control input to the performance variables. Often, only a single Markov parameter is required, even in the nonminimum-phase case. We demonstrate the algorithm on command-following and disturbance-rejection problems, where the command and disturbance spectra are unknown. This controller is based on a retrospective performance objective, where the controller is updated using either batch or recursive least squares.
\end{abstract}

\section{Introduction}

One of the ironies of feedback control is that the underlying motivation for feedback is uncertainty, yet uncertainty in the dynamics of a system can degrade performance and render the closed-loop system unstable. Although robust control can mitigate this difficulty by trading performance for stability, unpredictable changes that occur during operation may be sufficiently large so as to render prior uncertainty analysis unreliable.

Unlike robust control, an adaptive controller is self-tuned during operation. This tuning accounts for the actual - and possibly changing - dynamics of the system as well as the nature of the external signals, such as commands and disturbances. Adaptive control may also be required for systems that are difficult to model due to unknown physics or due to the inability to perform sufficiently accurate identification. Adaptive control may depend on prior modeling information, such as bounds on the model order and parameters, or it may entail explicit on-line identification. These approaches are known, respectively, as direct and indirect adaptive control. The key issue then becomes the nature of the modeling information required by the adaptive controller provided either prior to or during operation.

Whether the adaptive controller is direct or indirect, it is desirable to develop algorithms that require the least amount of modeling information. In the most extreme case, LQG control, which is nonadaptive, requires a complete and exact model of the plant dynamics as well as a complete and exact model of the disturbance and sensor noise statistics and spectrum. Although robust control techniques increase forgiveness to modeling errors, these methods require a nominal model as well as bounds on all uncertain parameters. These bounds can be determined by prior analysis or identification, but may become invalid during operation if changes occur, and, as noted above, they necessitate tradeoffs between stability and performance.

Minimizing the amount of modeling information needed for control is the motivation for adaptive control, which is inherently robust to modeling information that it does not require. For example, if an adaptive controller requires no knowledge of the plant pole locations, then it is unconditionally robust to the actual pole locations, assuming that they are constant or, perhaps, slowly changing. While the closed-loop system may depend on the actual locations of the poles, the achievable performance under adaptive control is not impeded by the lack of prior knowledge regarding that modeling information. The main benefit of adaptive control is thus the reduced need to model the system for controller tuning without sacrificing performance.

In many applications, modeling is performed for reasons other than controller tuning, such as for design studies and certification. However, modeling for control often requires knowledge of system properties that are not provided by models developed for other purposes. For example, bounds on the plant phase near

\footnotetext{
${ }^{*}$ This work was supported in part by NASA GSRP grant NNX09AO55H and IRAC grant NNX08AB92A.

$\dagger$ A. M. D’Amato, E. Dogan Sumer and D. S. Bernstein are with the Department of Aerospace Engineering, University of Michigan, Ann Arbor, MI, USA. \{ amdamato, dogan, dsbaero\}@umich.edu
} 
crossover directly affect stability. In addition, many applications require plant operation over a wide range of conditions that may lead to large changes in the dynamics of the system, for example, changes in modal frequencies and damping. The engineering cost of modeling a system over a wide range of operational conditions may be exorbitant, especially if ambient disturbances and test limitations make it impossible to identify a viable model.

The ultimate objective of adaptive control is thus to reduce - to the greatest possible extent - the reliance on modeling for the purpose of controller tuning. In fact, reducing the required modeling information can decrease the cost of modeling while increasing the reliability of the system to changes that occur during operation. These are the benefits of adaptive control.

In adaptive control, the controller is tuned to the actual plant during operation. However, this ability comes at a cost. Adaptive control algorithms may require restrictive assumptions, such as full-state feedback, positive realness, minimum-phase zeros, matched disturbances, as well as information on the sign of the high frequency gain, relative degree, or zero locations. ${ }^{1-4}$ In particular, the starting point for the present paper is the retrospective cost adaptive control (RCAC) approach. ${ }^{5-8}$ This direct adaptive control approach is applicable to MIMO (output feedback) plants that are possibly unstable and nonminimum phase (NMP) with uncertain command and disturbance spectra. The modeling information required by RCAC in ${ }^{5-8}$ is the first nonzero Markov parameter and locations of the NMP zeros, if any. Alternatively, a collection of Markov parameters can be used as long as a sufficient number is available to capture the NMP zero locations.

The present paper extends prior RCAC results by describing a modification of RCAC that does not require knowledge of the locations of the NMP zeros. Instead, this extension requires knowledge of a limited number of Markov parameters; typically only one Markov parameter is needed. The significant aspect of this extension is the fact that knowledge of the NMP zeros is not needed. This extension thus increases the applicability of the method to systems with unknown NMP zeros, as well as systems with NMP zeros that may be changing slowly due to aging or due to a slowly varying linearization of a nonlinear plant.

The algorithm developed in the present paper is demonstrated on a representative collection of SISO and MIMO examples. In all cases, the number of Markov parameters that are used is not sufficient to determine the NMP zeros of the system. Consequently, these examples demonstrate the ability to control MIMO NMP systems with unknown NMP zeros.

In the present paper we assume that that the Markov parameters that are used in RCAC are exactly known. However, in practice, these parameters are uncertain due to modeling errors. Therefore, in ${ }^{11}$ we consider the effect of uncertainty in the Markov parameters.

\section{Problem Formulation}

Consider the MIMO discrete-time system

$$
\begin{aligned}
x(k+1) & =A x(k)+B u(k)+D_{1} w(k), \\
y(k) & =C x(k)+D_{2} w(k), \\
z(k) & =E_{1} x(k)+E_{0} w(k),
\end{aligned}
$$

where $x(k) \in \mathbb{R}^{n}, y(k) \in \mathbb{R}^{l_{y}}, z(k) \in \mathbb{R}^{l_{z}}, u(k) \in \mathbb{R}^{l_{u}}, w(k) \in \mathbb{R}^{l_{w}}$, and $k \geq 0$. Our goal is to develop an adaptive output feedback controller that minimizes the performance variable $z$ in the presence of the exogenous signal $w$ with minimal modeling information about the dynamics and $w$. The block diagram for (1)-(3) is shown in Figure 1, where $G(\mathbf{q})=\left[G_{z w}(\mathbf{q}) G_{z u}(\mathbf{q})\right]$ and

$$
z(k)=G_{z w}(\mathbf{q}) w(k)+G_{z u}(\mathbf{q}) u(k),
$$

where $\mathbf{q}$ is the forward-shift operator. Note that $w$ can represent either a command signal to be followed, an external disturbance to be rejected, or both. The system (1)-(3) can represent a sampled-data application arising from a continuous-time system with sample and hold operations.

If $D_{1}=0$ and $E_{0} \neq 0$, then the objective is to have the output $E_{1} x$ follow the command signal $-E_{0} w$. On the other hand, if $D_{1} \neq 0$ and $E_{0}=0$, then the objective is to reject the disturbance $w$ from the performance measurement $E_{1} x$. Furthermore, if $D_{1}=\left[\begin{array}{cc}\hat{D}_{1} & 0\end{array}\right], E_{0}=\left[\begin{array}{ll}0 & \hat{E}_{0}\end{array}\right]$, and $w(k)=\left[w_{1}(k)^{\mathrm{T}} w_{2}(k)^{\mathrm{T}}\right]^{\mathrm{T}}$, then the objective is to have $E_{1} x$ follow the command $-\hat{E}_{0} w_{2}$ while rejecting the disturbance $w_{1}$. Lastly, if $D_{1}$ and $E_{0}$ are empty matrices, then the objective is output stabilization, that is, convergence of $z$ to zero. 


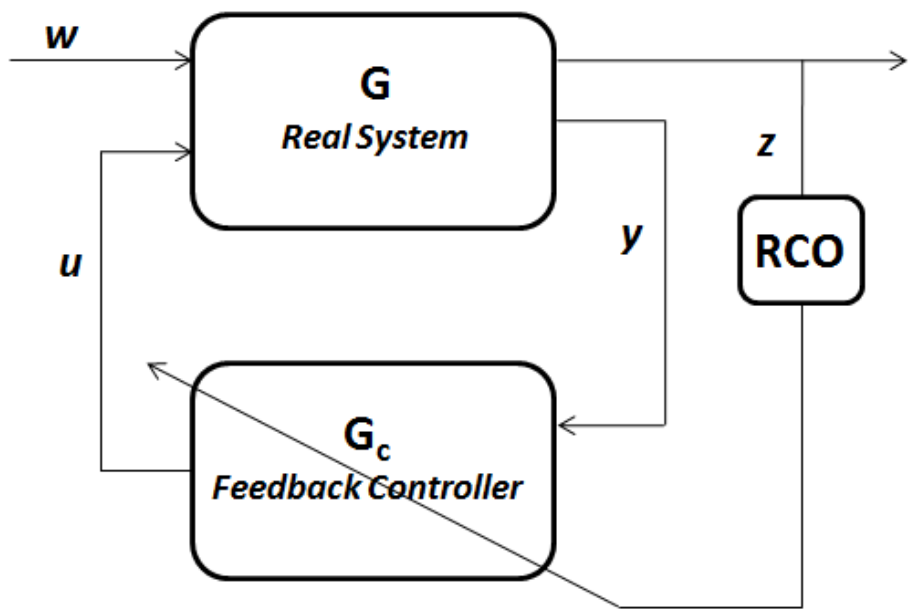

Figure 1. Disturbance-rejection and command-following architecture.

\section{Retrospective Surrogate Cost}

For $i \geq 1$, define the Markov parameter of $G_{z u}$ given by

$$
H_{i} \triangleq E_{1} A^{i-1} B
$$

For example, $H_{1}=E_{1} B$ and $H_{2}=E_{1} A B$. Let $r$ be a positive integer. Then, for all $k \geq r$,

$$
x(k)=A^{r} x(k-r)+\sum_{i=1}^{r} A^{i-1} B u(k-i)+\sum_{i=1}^{r} A^{i-1} D_{1} w(k-i),
$$

and thus

$$
z(k)=E_{1} A^{r} x(k-r)+\sum_{i=1}^{r} E_{1} A^{i-1} D_{1} w(k-i)+E_{0} w(k)+\bar{H} \bar{U}(k-1),
$$

where

$$
\bar{H} \triangleq\left[\begin{array}{lll}
H_{1} & \cdots & H_{r}
\end{array}\right] \in \mathbb{R}^{l_{z} \times r l_{u}}
$$

and

$$
\bar{U}(k-1) \triangleq\left[\begin{array}{c}
u(k-1) \\
\vdots \\
u(k-r)
\end{array}\right]
$$

Next, we rearrange the columns of $\bar{H}$ and the components of $\bar{U}(k-1)$ and partition the resulting matrix and vector so that

$$
\bar{H} \bar{U}(k-1)=\mathcal{H}^{\prime} U^{\prime}(k-1)+\mathcal{H} U(k-1),
$$

where $\mathcal{H}^{\prime} \in \mathbb{R}^{l_{z} \times\left(r l_{u}-l_{U}\right)}, \mathcal{H} \in \mathbb{R}^{l_{z} \times l_{U}}, U^{\prime}(k-1) \in \mathbb{R}^{r l_{u}-l_{U}}$, and $U(k-1) \in \mathbb{R}^{l_{U}}$. Then, we can rewrite $(7)$ as

$$
z(k)=\mathcal{S}(k)+\mathcal{H} U(k-1),
$$

where

$$
\mathcal{S}(k) \triangleq E_{1} A^{r} x(k-r)+\sum_{i=1}^{r} E_{1} A^{i-1} D_{1} w(k-i)+E_{0} w(k)+\mathcal{H}^{\prime} U^{\prime}(k-1) .
$$


For example, $\bar{H}=\left[\begin{array}{lllll}H_{1} & H_{2} & H_{3} & H_{4} & H_{5}\end{array}\right]$,

$$
\mathcal{H}^{\prime}=\left[\begin{array}{lll}
H_{1} & H_{2} & H_{4}
\end{array}\right], \quad U^{\prime}(k-1)=\left[\begin{array}{c}
u(k-1) \\
u(k-2) \\
u(k-4)
\end{array}\right],
$$

and

$$
\mathcal{H}=\left[\begin{array}{ll}
H_{3} & H_{5}
\end{array}\right], \quad U(k-1)=\left[\begin{array}{l}
u(k-3) \\
u(k-5)
\end{array}\right] .
$$

Next, for $j=1, \ldots, s$, we rewrite (9) with a delay of $k_{j}$ time steps, where $0 \leq k_{1} \leq k_{2} \leq \cdots \leq k_{s}$, in the form

$$
z\left(k-k_{j}\right)=\mathcal{S}_{j}\left(k-k_{j}\right)+\mathcal{H}_{j} U_{j}\left(k-k_{j}-1\right),
$$

where (10) becomes

$$
\mathcal{S}_{j}\left(k-k_{j}\right) \triangleq E_{1} A^{r} x\left(k-k_{j}-r\right)+\sum_{i=1}^{r} E_{1} A^{i-1} D_{1} w\left(k-k_{j}-i\right)+E_{0} w\left(k-k_{j}\right)+\mathcal{H}_{j}^{\prime} U_{j}^{\prime}\left(k-k_{j}-1\right)
$$

and (8) becomes

$$
\bar{H} \bar{U}\left(k-k_{j}-1\right)=\mathcal{H}_{j}^{\prime} U_{j}^{\prime}\left(k-k_{j}-1\right)+\mathcal{H}_{j} U_{j}\left(k-k_{j}-1\right),
$$

where $\mathcal{H}_{j}^{\prime} \in \mathbb{R}^{l_{z} \times\left(r l_{u}-l_{U_{j}}\right)}, \mathcal{H}_{j} \in \mathbb{R}^{l_{z} \times l_{U_{j}}}, U_{j}^{\prime}\left(k-k_{j}-1\right) \in \mathbb{R}^{r l_{u}-l_{U_{j}}}$, and $U_{j}\left(k-k_{j}-1\right) \in \mathbb{R}^{l_{U_{j}}}$. Now, by stacking $z\left(k-k_{1}\right), \ldots, z\left(k-k_{s}\right)$, we define the extended performance

$$
Z(k) \triangleq\left[\begin{array}{c}
z\left(k-k_{1}\right) \\
\vdots \\
z\left(k-k_{s}\right)
\end{array}\right] \in \mathbb{R}^{s l_{z}}
$$

Therefore,

$$
Z(k) \triangleq \tilde{\mathcal{S}}(k)+\tilde{\mathcal{H}} \tilde{U}(k-1)
$$

where

$$
\tilde{\mathcal{S}}(k) \triangleq\left[\begin{array}{c}
\mathcal{S}_{1}\left(k-k_{1}\right) \\
\vdots \\
\mathcal{S}_{s}\left(k-k_{s}\right)
\end{array}\right] \in \mathbb{R}^{s l_{z}},
$$

$\tilde{U}(k-1)$ has the form

$$
\tilde{U}(k-1) \triangleq\left[\begin{array}{c}
u\left(k-q_{1}\right) \\
\vdots \\
u\left(k-q_{l_{\tilde{U}}}\right)
\end{array}\right] \in \mathbb{R}^{l_{\tilde{U}}},
$$

where, for $i=1, \ldots, l_{\tilde{U}}, k_{1} \leq q_{i} \leq k_{s}+r$, and $\tilde{\mathcal{H}} \in \mathbb{R}^{s l_{z} \times l_{\tilde{U}}}$ is constructed according to the structure of $\tilde{U}(k-1)$. The vector $\tilde{U}(k-1)$ is formed by stacking $U_{1}\left(k-k_{1}-1\right), \ldots, U_{s}\left(k-k_{s}-1\right)$ and removing copies of repeated components.

For example, with $k_{1}=0$ and $k_{2}=1$, stacking $U_{1}(k-1)=\left[\begin{array}{c}u(k-1) \\ u(k-2)\end{array}\right]$ and $U_{2}(k-2)=u(k-2)$ results in $\tilde{U}(k-1)=\left[\begin{array}{l}u(k-1) \\ u(k-2)\end{array}\right]$. The coefficient matrix $\tilde{\mathcal{H}}$ consists of the entries of $\mathcal{H}_{1}, \ldots, \mathcal{H}_{s}$ arranged according to the structure of $\tilde{U}(k-1)$. 
Next, we define the surrogate performance

$$
\hat{z}\left(k-k_{j}\right) \triangleq \mathcal{S}_{j}\left(k-k_{j}\right)+\mathcal{H}_{j} \hat{U}_{j}\left(k-k_{j}-1\right),
$$

where the past controls $U_{j}\left(k-k_{j}-1\right)$ in (11) are replaced by the surrogate controls $\hat{U}_{j}\left(k-k_{j}-1\right)$. In analogy with (13), the extended surrogate performance for (17) is defined as

$$
\hat{Z}(k) \triangleq\left[\begin{array}{c}
\hat{z}\left(k-k_{1}\right) \\
\vdots \\
\hat{z}\left(k-k_{s}\right)
\end{array}\right] \in \mathbb{R}^{s l_{z}}
$$

and thus is given by

$$
\hat{Z}(k)=\tilde{\mathcal{S}}(k)+\tilde{\mathcal{H}} \hat{\tilde{U}}(k-1),
$$

where the components of $\hat{\tilde{U}}(k-1) \in \mathbb{R}^{l} \tilde{U}$ are the components of $\hat{U}_{1}\left(k-k_{1}-1\right), \ldots, \hat{U}_{s}\left(k-k_{s}-1\right)$ ordered in the same way as the components of $\tilde{U}(k-1)$. Subtracting (14) from (19) yields

$$
\hat{Z}(k)=Z(k)-\tilde{\mathcal{H}} \tilde{U}(k-1)+\tilde{\mathcal{H}} \tilde{\tilde{U}}(k-1) .
$$

Finally, we define the retrospective cost function

$$
J(\hat{\tilde{U}}(k-1), k) \triangleq \hat{Z}^{\mathrm{T}}(k) R(k) \hat{Z}(k),
$$

where $R(k) \in \mathbb{R}^{l_{z} s \times l_{z} s}$ is a positive-definite performance weighting. The goal is to determine refined controls $\hat{\tilde{U}}(k-1)$ that would have provided better performance than the controls $U(k)$ that were applied to the system. The refined control values $\hat{\tilde{U}}(k-1)$ are subsequently used to update the controller.

\section{Cost Function Optimization with Adaptive Regularization}

To ensure that (21) has a global minimizer, we consider the regularized cost

$$
\begin{aligned}
\bar{J}(\hat{\tilde{U}}(k-1), k) & \triangleq \hat{Z}^{\mathrm{T}}(k) R(k) \hat{Z}(k) \\
& +\eta(k) \hat{\tilde{U}}^{\mathrm{T}}(k-1) \hat{\tilde{U}}(k-1),
\end{aligned}
$$

where $\eta(k) \geq 0$. Substituting (20) into (22) yields

$$
\begin{aligned}
\bar{J}(\hat{\tilde{U}}(k-1), k) & =\hat{\tilde{U}}(k-1)^{\mathrm{T}} \mathcal{A}(k) \hat{\tilde{U}}(k-1) \\
& +\mathcal{B}(k) \hat{\tilde{U}}(k-1)+\mathcal{C}(k),
\end{aligned}
$$

where

$$
\begin{aligned}
& \mathcal{A}(k) \triangleq \tilde{\mathcal{H}}^{\mathrm{T}} R(k) \tilde{\mathcal{H}}+\eta(k) I_{l} \\
& \mathcal{B}(k) \triangleq 2 \tilde{\mathcal{H}}^{\mathrm{T}} R(k)[Z(k)-\tilde{\mathcal{H}} \tilde{U}(k-1)], \\
& \mathcal{C}(k) \triangleq Z^{\mathrm{T}}(k) R(k) Z(k)-2 Z^{\mathrm{T}}(k) R(k) \tilde{\mathcal{H}} \tilde{U}(k-1)+\tilde{U}^{\mathrm{T}}(k-1) \tilde{\mathcal{H}}^{\mathrm{T}} R(k) \tilde{\mathcal{H}} \tilde{U}(k-1) .
\end{aligned}
$$

If either $\tilde{\mathcal{H}}$ has full column rank or $\eta(k)>0$, then $\mathcal{A}(k)$ is positive definite. In this case, $\bar{J}(\hat{\tilde{U}}(k-1), k)$ has the unique global minimizer

$$
\hat{\tilde{U}}(k-1)=-\frac{1}{2} \mathcal{A}^{-1}(k) \mathcal{B}(k)
$$




\section{Controller Construction}

The control $u(k)$ is given by the strictly proper time-series controller of order $n_{\mathrm{c}}$ given by

$$
u(k)=\sum_{i=1}^{n_{\mathrm{c}}} M_{i}(k) u(k-i)+\sum_{i=1}^{n_{\mathrm{c}}} N_{i}(k) y(k-i),
$$

where, for all $i=1, \ldots, n_{\mathrm{c}}, M_{i}(k) \in \mathbb{R}^{l_{u} \times l_{u}}$ and $N_{i}(k) \in \mathbb{R}^{l_{u} \times l_{y}}$. The control (28) can be expressed as

$$
u(k)=\theta(k) \phi(k-1),
$$

where

$$
\theta(k) \triangleq\left[M_{1}(k) \cdots M_{n_{\mathrm{c}}}(k) N_{1}(k) \cdots N_{n_{\mathrm{c}}}(k)\right] \in \mathbb{R}^{l_{u} \times n_{\mathrm{c}}\left(l_{u}+l_{z}\right)}
$$

and

$$
\phi(k-1) \triangleq\left[\begin{array}{c}
u(k-1) \\
\vdots \\
u\left(k-n_{\mathrm{c}}\right) \\
y(k-1) \\
\vdots \\
y\left(k-n_{\mathrm{c}}\right)
\end{array}\right] \in \mathbb{R}^{n_{\mathrm{c}}\left(l_{u}+l_{y}\right)} .
$$

Let $d$ be a positive integer such that $\tilde{U}(k-1)$ contains $u(k-d)$, and let $p$ be the data window size. Then $\hat{\tilde{U}}(k-1), \ldots, \hat{\tilde{U}}(k-p+1)$ contain $\hat{u}(k-d), \ldots, \hat{u}(k-d+1-p)$, respectively. We can thus construct

$$
\Psi_{p}(k-d) \triangleq\left[\begin{array}{c}
\hat{u}^{\mathrm{T}}(k-d) \\
\vdots \\
\hat{u}^{\mathrm{T}}(k-d+1-p)
\end{array}\right] \in \mathbb{R}^{p \times l_{u}} .
$$

The matrix $\Psi_{p}(k-d)$ is used below for the controller update.

\section{A. Batch Least Squares Update of $\theta(k)$}

Define

$$
\Phi_{p}(k-d-1) \triangleq\left[L_{y, p}(k-d-1) \quad L_{u, p}(k-d-1)\right] \in \mathbb{R}^{p \times\left[n_{c}\left(l_{u}+l_{y}\right)\right]}
$$

where

$$
L_{y, p}(k-d-1) \triangleq\left[\begin{array}{ccc}
y(k-d-1) & \cdots & y\left(k-n_{c}-d\right) \\
\vdots & \ddots & \vdots \\
y(k-d-p-1) & \cdots & y\left(k-n_{c}-d-p\right)
\end{array}\right]
$$

and

$$
L_{u, p}(k-d-1) \triangleq\left[\begin{array}{ccc}
u(k-d-1) & \cdots & u\left(k-n_{c}-d\right) \\
\vdots & \ddots & \vdots \\
u(k-d-p-1) & \cdots & u\left(k-n_{c}-d-p\right)
\end{array}\right] .
$$

Next, consider the quadratic cost

$$
J_{\mathrm{B}}(\theta(k)) \triangleq\left\|\Phi_{p}(k-d-1) \theta^{\mathrm{T}}(k)-\Psi_{p}(k-d)\right\|^{2}+\alpha(k) \operatorname{tr}\left[\theta(k) \theta^{\mathrm{T}}(k)\right],
$$

where $\alpha(k)>0$. Minimizing (36) yields the controller update

$$
\theta^{\mathrm{T}}(k)=\left[\Phi_{p}^{\mathrm{T}}(k-d-1) \Phi_{p}(k-d-1)+\alpha(k) I\right]^{-1} \Phi_{p}^{\mathrm{T}}(k-d-1) \Psi_{p}(k-d) .
$$




\section{B. Recursive Least Squares Update of $\theta(k)$}

Next, noting that $\Psi_{1}(k-d)=\hat{u}^{\mathrm{T}}(k-d)$, we define the cumulative cost function

$$
J_{\mathrm{R}}(\theta(k)) \triangleq \sum_{i=d+1}^{k} \lambda^{k-i}\left\|\phi^{\mathrm{T}}(i-d-1) \theta^{\mathrm{T}}(i-1)-\hat{u}^{\mathrm{T}}(i-d)\right\|^{2},
$$

where $\|\cdot\|$ is the Euclidean norm, and $\lambda(k) \in(0,1]$ is the forgetting factor. Minimizing (38) yields

$$
\begin{aligned}
\theta^{\mathrm{T}}(k) \triangleq & \theta^{\mathrm{T}}(k-1)+P(k-1) \phi(k-d-1)\left[\phi^{\mathrm{T}}(k-d) P(k-1) \phi(k-d-1)+\lambda(k)\right]^{-1} \\
\cdot & {\left[\phi^{\mathrm{T}}(k-d-1) \theta^{\mathrm{T}}(k-1)-\hat{u}^{\mathrm{T}}(k-d)\right] . }
\end{aligned}
$$

The error covariance is updated by

$$
\begin{aligned}
P(k) \triangleq & \lambda^{-1}(k) P(k-1)-\lambda^{-1}(k) P(k-1) \phi(k-d-1)\left[\phi^{\mathrm{T}}(k-d-1) P(k-1) \phi(k-d)+\lambda(k)\right]^{-1} \\
& \cdot \phi^{\mathrm{T}}(k-d-1) P(k-1) .
\end{aligned}
$$

We initialize the error covariance matrix as $P(0)=\gamma I$, where $\gamma>0$.

\section{Stability Analysis}

\section{A. Conditions for Convergence of $z(k)-\hat{z}(k)$ to Zero}

Consider the retrospective system

$$
\begin{aligned}
\hat{x}(k+1) & =A x(k)+B \hat{u}(k)+D_{1} w(k), \\
\hat{z}(k) & =E_{1} \hat{x}(k)+E_{0} w(k),
\end{aligned}
$$

which is obtained by replacing $u(k)$ in (1) with $\hat{u}(k)$. The extended retrospective system is given by

$$
\begin{aligned}
\hat{X}(k+1) & =\tilde{A} X(k)+\tilde{B} \hat{\tilde{U}}(k)+\tilde{B}^{\prime} \hat{\tilde{U}}^{\prime}(k)+\tilde{D}_{1} W(k), \\
\hat{Z}(k) & =\tilde{E}_{1} \hat{X}(k)+\tilde{E}_{0} W(k),
\end{aligned}
$$

where

$$
\begin{gathered}
\hat{X}(k)=\left[\begin{array}{c}
\hat{x}\left(k-k_{1}\right) \\
\vdots \\
\hat{x}\left(k-k_{s}\right)
\end{array}\right] \in \mathbb{R}^{s n}, \\
W(k)=\left[\begin{array}{c}
w\left(k-k_{1}\right) \\
\vdots \\
w\left(k-k_{s}\right)
\end{array}\right] \in \mathbb{R}^{s l_{w},} \\
\tilde{U}^{\prime}(k-1) \triangleq\left[\begin{array}{c}
u\left(k-q_{1}^{\prime}\right) \\
\vdots \\
u\left(k-q_{\tilde{U}^{\prime}}^{\prime}\right)
\end{array}\right] \in \mathbb{R}^{l} \tilde{U}^{\prime},
\end{gathered}
$$

$\tilde{A} \triangleq I_{s} \otimes A \in \mathbb{R}^{s n \times s n}, \tilde{D}_{1} \triangleq I_{s} \otimes D_{1} \in \mathbb{R}^{s n \times s l_{w}}, \tilde{E}_{0} \triangleq I_{s} \otimes E_{1} \in \mathbb{R}^{s l_{z} \times s l_{w}}, \tilde{E}_{1} \triangleq I_{s} \otimes E_{1} \in \mathbb{R}^{s l_{z} \times s n}$, and $\otimes$ is the Kronecker product. The matrices $\tilde{B} \in \mathbb{R}^{s n \times l_{\tilde{U}}}$ and $\tilde{B}^{\prime} \in \mathbb{R}^{s n \times l_{\tilde{U}}^{\prime}}$ are block-row matrices with block entries $B$ and $0_{n \times l_{u}}$ such that

$$
\tilde{B} \hat{\tilde{U}}(k)+\tilde{B}^{\prime} \hat{\tilde{U}}^{\prime}(k)=\left[\begin{array}{c}
B \hat{u}\left(k-k_{1}\right) \\
\vdots \\
B \hat{u}\left(k-k_{s}\right)
\end{array}\right] \in \mathbb{R}^{s l_{u}},
$$


where $\hat{\tilde{U}}^{\prime}(k)$ is formed by replacing the entries $u\left(k-q_{i}^{\prime}\right)$ of $\tilde{U}^{\prime}(k)$ by $\hat{u}\left(k-q_{i}^{\prime}\right)$ for $i=1, \ldots, l_{\tilde{U}^{\prime}}$.

The following result gives conditions under which $\hat{Z}(k)=0$.

Fact VI.1 Assume that $\tilde{\mathcal{H}}$ has full column rank, $\eta(k)=0, R(k)=I$, and $Z(k)$ is in the range of $\tilde{\mathcal{H}}$ for all $k$, and let $\hat{\tilde{U}}(k-1)$ be given by $(27)$. Then $\hat{Z}(k)=0$.

Proof. Since $Z(k)$ is in the range of $\tilde{\mathcal{H}}$, there exists $Q \in \mathbb{R}^{s l \tilde{u}}$ such that $Z(k)=\tilde{\mathcal{H}} Q$. Substituting $(27)$ into (20) yields

$$
\begin{aligned}
\hat{Z}(k) & =Z(k)+\tilde{\mathcal{H}}\left(\tilde{\mathcal{H}}^{\mathrm{T}} \tilde{\mathcal{H}}\right)^{-1} \tilde{\mathcal{H}}^{\mathrm{T}}(-Z(k)+\tilde{\mathcal{H}} \tilde{U})-\tilde{\mathcal{H}} \tilde{U} \\
& =Z(k)-\tilde{\mathcal{H}}\left(\tilde{\mathcal{H}}^{\mathrm{T}} \tilde{\mathcal{H}}\right)^{-1} \tilde{\mathcal{H}}^{\mathrm{T}} Z(k) \\
& =\tilde{\mathcal{H}} Q-\tilde{\mathcal{H}}\left(\tilde{\mathcal{H}}^{\mathrm{T}} \tilde{\mathcal{H}}\right)^{-1} \tilde{\mathcal{H}}^{\mathrm{T}} \tilde{\mathcal{H}} Q=0 .
\end{aligned}
$$

The next result assumes that the recursive-least-squares optimization yields $u(k-d)-\hat{u}(k-d) \rightarrow 0$ as $k \rightarrow \infty$, that is, $\theta(k) \phi(k-d-1)-\hat{u}(k-d) \rightarrow \infty$ as $k \rightarrow \infty$.

Fact VI.2 Assume that $\theta(k)$ is updated using (39) and (40), and assume that $\theta(k) \phi(k-d-1)-\hat{u}(k-d) \rightarrow$ 0 as $k \rightarrow \infty$. Then $x(k)-\hat{x}(k) \rightarrow 0$ as $k \rightarrow \infty$.

Proof. It follows from (1) and (41) that

$$
x(k-d+1)-\hat{x}(k-d+1)=B u(k-d)-B \hat{u}(k-d) .
$$

It follows from $(29)$ that $u(k-d)=\theta(k-d) \phi(k-d-1)$. Defining $g(k) \triangleq \theta(k) \phi(k-d-1)-\hat{u}(k-d),(49)$ becomes

$$
x(k-d+1)-\hat{x}(k-d+1)=B[\theta(k-d)-\theta(k)] \phi(k-d-1)+B g(k) .
$$

Since $g(k) \rightarrow 0$ as $k \rightarrow \infty$, it follows from (39) that $\theta(k)-\theta(k-1) \rightarrow 0$ as $k \rightarrow \infty$. It thus follows from (50) that $x(k-d+1)-\hat{x}(k-d+1) \rightarrow 0$ as $k \rightarrow \infty$.

In view of Fact VI.2, we assume henceforth that $k$ is sufficiently large that the difference between $\hat{x}(k)$, $\hat{u}(k), \hat{y}(k)$, and $\hat{z}(k)$ and $x(k), u(k), y(k)$, and $z(k)$, respectively, is negligible. For convenience we set $d=r$. The following analysis focuses on the subsequent behavior of $\hat{x}(k), \hat{u}(k)$, and $\hat{z}(k)$, when $\eta(k)=0$ and $R(k)=I$.

\section{B. Boundedness of the Internal State}

Next, we introduce the ideal system performance

$$
z^{*}(k)=E_{1} A^{r} x^{*}(k-r)+\sum_{i=1}^{r} E_{1} A^{i-1} D_{1} w(k-i)+E_{0} w(k)+\mathcal{H}^{\prime} U^{\prime}(k-1)+\mathcal{H} U^{*}(k-1),
$$

where $x^{*}(k)$ is the state of the ideal system and $U^{*}(k-1)$ is defined analogously to $U(k-1)$, with $u(k)$ replaced by $u^{*}(k)$, where

$$
\begin{gathered}
u^{*}(k)=\theta^{*} \phi^{*}(k-1), \\
\phi^{*}(k-1) \triangleq\left[\begin{array}{c}
u^{*}(k-1) \\
\vdots \\
u^{*}\left(k-n_{\mathrm{c}}\right) \\
y^{*}(k-1) \\
\vdots \\
y^{*}\left(k-n_{\mathrm{c}}\right)
\end{array}\right],
\end{gathered}
$$


and the ideal controller $\theta^{*}$ is assumed to yield the ideal performance

$$
z^{*}(k) \equiv 0 .
$$

Adding and subtracting $E_{1} A^{r} \hat{x}(k-r)$ to and from (51) yields

$$
z^{*}(k)=\mathcal{S}(k)+E_{1} A^{r} e(k-r)+\mathcal{H} U^{*}(k-1),
$$

where $\mathcal{S}(k)$ is defined by (10) with $x(k)$ replaced by $\hat{x}(k)$, and $e(k) \triangleq x^{*}(k)-\hat{x}(k)$.

The extended ideal system is given by

$$
\begin{aligned}
X^{*}(k+1) & =\tilde{A} X^{*}(k)+\tilde{B} \tilde{U}^{*}(k)+\tilde{B} \tilde{U}^{\prime}(k)+\tilde{D}_{1} W(k), \\
Z^{*}(k) & =\tilde{\mathcal{S}}(k)+\tilde{E}_{1} \tilde{A}^{r} E(k-1)+\tilde{\mathcal{H}} \tilde{U}^{*}(k-1)=0,
\end{aligned}
$$

where $X^{*}(k+1)$ and $Z^{*}(k)$ are defined in the same way as $X(k+1)$ and $Z(k), E(k) \triangleq X^{*}(k)-\hat{X}(k)$, and

$$
\begin{gathered}
\tilde{U}^{*}(k) \triangleq\left[I_{l_{\tilde{U}}} \otimes \theta^{*}\right] \tilde{\phi}^{*}(k-1), \\
\tilde{\phi}^{*}(k) \triangleq\left[\begin{array}{c}
\phi^{*}\left(k-q_{1}\right) \\
\vdots \\
\phi^{*}\left(k-q_{l_{\tilde{U}}}\right)
\end{array}\right] .
\end{gathered}
$$

The goal is to drive the refined controls $\hat{\tilde{U}}(k-1)$ to $\tilde{U}^{*}(k-1)$ to ensure that $\theta(k)-\theta^{*} \rightarrow 0$ as $k \rightarrow \infty$.

Next, subtracting (19) from (57) and solving for $\hat{\tilde{U}}(k-1)$ yields

$$
\hat{\tilde{U}}(k-1)=\tilde{\mathcal{H}}^{\dagger}\left[\tilde{E}_{1} \tilde{A}^{r} E(k-1)+\tilde{\mathcal{H}} \tilde{U}^{*}(k-1)+\hat{Z}(k)\right],
$$

where $\tilde{\mathcal{H}}^{\dagger} \tilde{\mathcal{H}}=I_{l_{\tilde{U}}}$ and $\tilde{\mathcal{H}}$ is assumed to have full column rank.

Under the assumptions of Fact VI.1, $\hat{Z}(k)=0$ and therefore (60) reduces to

$$
\hat{\tilde{U}}(k-1)=\tilde{\mathcal{H}}^{\dagger} \tilde{E}_{1} \tilde{A}^{r} E(k-1)+\tilde{U}^{*}(k-1) .
$$

Subtracting (43) from (56), and using (61) yields the error dynamics

$$
E(k)=\left(\tilde{A}-\tilde{B} \tilde{\mathcal{H}}^{\dagger} \tilde{E}_{1} \tilde{A}^{r}\right) E(k-1) .
$$

Therefore, if $\tilde{A}-\tilde{B} \tilde{\mathcal{H}}^{\dagger} \tilde{E}_{1} \tilde{A}^{r}$ is asymptotically stable, then $x(k)-x^{*}(k) \rightarrow 0$ as $k \rightarrow \infty$. Furthermore, $z(k)-z^{*}(k)=E_{1} x(k)-E_{1} x^{*}(k) \rightarrow 0$ as $k \rightarrow \infty$. Since $z^{*}(k)=E_{1} x^{*}(k)=0$, it follows that $z(k) \rightarrow 0$ as $k \rightarrow \infty$.

\section{Illustrative Example}

Consider the case $d=r=s=1, \tilde{\mathcal{H}}^{\prime}=0_{l_{z} \times l_{u}}$, and $\tilde{\mathcal{H}}=H_{1}=E_{1} B$. Furthermore, assume that $H_{1}$ is square and invertible. Next, let

$$
G_{z u}(\mathbf{q})=E_{1}(\mathbf{q} I-A)^{-1} B
$$

and it follows from (4) that

$$
z(k)=\mathbf{q} G_{z u}(\mathbf{q}) u(k-1)+G_{z w}(\mathbf{q}) w(k) .
$$

Substituting (7) in (27), with $\eta(k)=0$, yields

$$
\hat{u}(k-1)=-H_{1}^{-1}\left[E_{1} A x(k-1)+E_{1} D_{1} w(k-1)\right],
$$


which yields the closed-loop retrospective system

$$
\begin{aligned}
& x(k)=\left(A-B H_{1}^{-1} E_{1} A\right) x(k-1)+\left(D_{1}-B H_{1}^{-1} E_{1} D_{1}\right) w(k-1), \\
& z(k)=E_{1} x(k) .
\end{aligned}
$$

Note that substituting (66) into (67) yields $\hat{z}(k)=0$. Next, for this example, the error system (62) is

$$
E(k+1)=\left(A-B H_{1}^{-1} E_{1} A\right) E(k) .
$$

The following result shows that the stability of (68) depends on the transmission zeros of $\left(A, B, E_{1}\right)$.

Fact VI.3 Assume that $l_{z}=l_{u}$ and $H_{1}$ is nonsingular. Then $A-B H_{1}^{-1} E_{1} A$ is asymptotically stable if and only if $\left(A, B, E_{1}\right)$ is minimum phase.

Proof. It follows from (63) that

$$
\begin{aligned}
\mathbf{q} G_{z u}(\mathbf{q}) & =E_{1} A(\mathbf{q} I-A)^{-1} B+H_{1} \\
& =\frac{1}{\alpha(\mathbf{q})} \beta(\mathbf{q})
\end{aligned}
$$

where $\alpha(\mathbf{q}) \in \mathbb{R}[\mathbf{q}]$ and $\beta(\mathbf{q}) \in \mathbb{R}^{l_{z} \times l_{z}}[\mathbf{q}]$ are defined by

$$
\begin{aligned}
\alpha(\mathbf{q}) & \triangleq \operatorname{det}(\mathbf{q} I-A) \\
& =\mathbf{q}^{n}+\alpha_{1} \mathbf{q}^{n-1}+\cdots+\alpha_{n-1} \mathbf{q}+\alpha_{n}, \\
\beta(\mathbf{q}) & \triangleq E_{1} \operatorname{Aadj}(\mathbf{q} I-A)+\alpha(\mathbf{q}) H_{1} \\
& =\mathbf{q}^{n} \beta_{0}+\mathbf{q}^{n-1} \beta_{1}+\cdots+\mathbf{q}^{2} \beta_{n-2}+\mathbf{q} \beta_{n-1} .
\end{aligned}
$$

Furthermore, note that

$$
\operatorname{det} \mathbf{q} G_{z u}(\mathbf{q})=\frac{\operatorname{det} \beta(\mathbf{q})}{\alpha^{l_{z}}(\mathbf{q})} .
$$

Since $H_{1}$ is nonsingular, it follows that $\mathbf{q} G_{z u}(\mathbf{q})$ has full normal rank, det $\beta(\mathbf{q})$ is not the zero polynomial, and the transmission zeros of $\mathbf{q} G_{z u}(\mathbf{q})$ are the roots of $\operatorname{det} \beta(\mathbf{q})$. Hence $(70)$ implies that

$$
\mathbf{q}^{-1} G_{z u}^{-1}(\mathbf{q})=\frac{\alpha(\mathbf{q})}{\operatorname{det} \beta(\mathbf{q})} \operatorname{adj} \beta(\mathbf{q}) .
$$

From (66), it follows that $\operatorname{det} \beta(\mathbf{q})=\operatorname{det}\left(\mathbf{q} I-A+B H_{1}^{-1} E_{1} A\right)$. Therefore, the eigenvalues of $A-B H_{1}^{-1} E_{1} A$ are the roots of $\operatorname{det} \beta(\mathbf{q})$. Consequently, $\left(A, B, E_{1}\right)$ is minimum phase if and only if $\mathbf{q}^{-1} G_{z u}^{-1}(\mathbf{q})$ is asymptotically stable.

Fact VI.4 Assume that $l_{z}=l_{u}<n$ and $H_{1}$ is nonsingular. If $\left(A-B H_{1}^{-1} E_{1} A, E_{1}\right)$ is detectable, then $\left(A, B, E_{1}\right)$ is minimum phase.

Proof. To prove necessity, define

$$
\mathcal{O} \triangleq\left[\begin{array}{c}
E_{1} \\
E_{1}\left(A-B H_{1}^{-1} E_{1} A\right) \\
\vdots \\
E_{1}\left(A-B H_{1}^{-1} E_{1} A\right)^{n-1}
\end{array}\right],
$$

which is the observability matrix of $\left(A-B H_{1}^{-1} E_{1} A, B H_{1}^{-1}, E_{1}\right)$. Since $H_{1}=E_{1} B$, it follows that $E_{1}(A-$ $\left.B H_{1}^{-1} E_{1} A\right)=0$. Therefore,

$$
\mathcal{O} \triangleq\left[\begin{array}{c}
E_{1} \\
0_{l_{z} \times n} \\
\vdots \\
0_{l_{z} \times n}
\end{array}\right]
$$

and thus, rank $\mathcal{O}=l_{z}$. Since $\left(A, B, E_{1}\right)$ is minimum phase it follows from Fact VI.3 that $A-B H_{1}^{-1} E_{1} A$ is asymptotically stable, and thus $\left(A-B H_{1}^{-1} E_{1} A, E_{1}\right)$ is detectable. 


\section{Regularized Retrospective Cost}

We now let $\eta(k)>0$. In this case, choosing $\hat{\tilde{U}}(k-1)$ as in $(27)$ yields

$$
\left.\hat{Z}(k)=Z(k)-\tilde{\mathcal{H}} \tilde{U}(k-1)+\tilde{\mathcal{H}}\left(\tilde{\mathcal{H}}^{\mathrm{T}} R(k) \tilde{\mathcal{H}}+\eta(k) I_{l}\right)^{-1} \tilde{\mathcal{H}}^{\mathrm{T}} R(k)[-Z(k)+\tilde{\mathcal{H}} \tilde{U}(k-1)]\right) .
$$

The following result is an extension of Fact VI.1, where we no longer assume that $\eta(k)=0$.

Fact VII.1 Assume that $\tilde{\mathcal{H}}$ has full column rank, $Z(k)$ is in the range of $\tilde{\mathcal{H}}$ for all $k, u(k)-\hat{u}(k) \rightarrow 0$ as $k \rightarrow \infty$, and let $\hat{\tilde{U}}(k-1)$ be given by $(27)$. Then $Z(k)-\hat{Z}(k) \rightarrow 0$ as $k \rightarrow \infty$.

Proof. Since $u(k)-\hat{u}(k) \rightarrow 0$ as $k \rightarrow \infty$, it follows that $\hat{\tilde{U}}(k)-\tilde{U}(k) \rightarrow 0$ as $k \rightarrow \infty$. Next, the retrospective cost function is

$$
\hat{Z}(k)=Z(k)-\tilde{\mathcal{H}}(\hat{\tilde{U}}(k)-\tilde{U}(k)),
$$

therefore, $\hat{Z}(k)-Z(k) \rightarrow 0$ as $k \rightarrow \infty$.

In view of Fact VII.1, we assume henceforth that $k$ is sufficiently large that the difference between $\hat{x}(k)$, $\hat{u}(k), \hat{y}(k)$, and $\hat{z}(k)$ and $x(k), u(k), y(k)$, and $z(k)$, respectively, is negligible. For convenience we set $d=r$. The following analysis focuses on the subsequent behavior of $\hat{x}(k)$ and $\hat{z}(k)$, when $\eta(k)>0$.

Substituting (27) into (43) yields

$$
\begin{aligned}
\hat{X}(k)=\tilde{A} \hat{X}(k) & +\tilde{B}\left(\tilde{\mathcal{H}}^{\mathrm{T}} R(k) \tilde{\mathcal{H}}+\eta(k-1) I_{l_{\tilde{U}}}\right)^{-1} \tilde{\mathcal{H}}^{\mathrm{T}} R(k)[-\hat{Z}(k)+\tilde{\mathcal{H}} \hat{\tilde{U}}(k-1)] \\
& +\tilde{B}^{\prime} \hat{\tilde{U}}^{\prime}(k-1)+\tilde{D}_{1} W(k-1), \\
\hat{Z}(k) & =\tilde{E}_{1} \hat{X}(k)+\tilde{E}_{0} W(k) .
\end{aligned}
$$

Next, we write the performance as

$$
\hat{Z}(k)=\tilde{E}_{1} \tilde{A}^{r} \hat{X}(k-1)+\tilde{\mathcal{H}} \hat{\tilde{U}}(k-1)+\tilde{\mathcal{H}}^{\prime} \hat{\tilde{U}}^{\prime}(k-1)+\tilde{D} \tilde{A}^{r} W(k-1) .
$$

Substituting (81) into (79) yields

$$
\begin{aligned}
\hat{X}(k)=[\tilde{A} & \left.-\tilde{B}\left(\tilde{\mathcal{H}}^{\mathrm{T}} R(k) \tilde{\mathcal{H}}+\eta(k-1) I_{l_{\tilde{U}}}\right)^{-1} \cdot \tilde{\mathcal{H}}^{\mathrm{T}} R(k) \tilde{E}_{1} \tilde{A}^{r}\right] \hat{X}(k-1), \\
& +\left[\tilde{D}_{1}-\tilde{B}\left(\tilde{\mathcal{H}}^{\mathrm{T}} R(k) \tilde{\mathcal{H}}+\eta(k-1) I_{\tilde{U}_{\tilde{U}}}\right)^{-1} \cdot \tilde{\mathcal{H}}^{\mathrm{T}} R(k) \tilde{D} \tilde{A}^{r}\right] W(k-1) \\
& +\left[\tilde{B}-\tilde{B}\left(\tilde{\mathcal{H}}^{\mathrm{T}} R(k) \tilde{\mathcal{H}}+\eta(k-1) I_{l_{\tilde{U}}}\right)^{-1} \tilde{\mathcal{H}}^{\mathrm{T}} R(k) \tilde{\mathcal{H}}^{\prime}\right] \hat{\tilde{U}}^{\prime}(k-1) .
\end{aligned}
$$

Therefore, it follows from (82) that if $\tilde{A}-\tilde{B}\left(\tilde{\mathcal{H}}^{\mathrm{T}} R(k) \tilde{\mathcal{H}}+\eta(k-1) I_{l_{\tilde{U}}}\right)^{-1} \tilde{\mathcal{H}}^{\mathrm{T}} R(k) \tilde{E}_{1} \tilde{A}^{r}$ is asymptotically stable, then $\hat{X}(k)$ and $Z(k)$ are bounded. Furthermore, note that $\tilde{A}-\tilde{B}\left(\tilde{\mathcal{H}}^{\mathrm{T}} R(k) \tilde{\mathcal{H}}+\eta(k-1) I_{l_{\tilde{U}}}\right)^{-1} \tilde{\mathcal{H}}^{\mathrm{T}} R(k) \tilde{E}_{1} \tilde{A}^{r} \rightarrow$ $\tilde{A}$ as $\eta(k) \rightarrow \infty$.

\section{Numerical Examples}

For all numerical examples in this paper we use the recursive least squares update (39) and (40). Furthermore, we consider only the disturbance rejection problem, where $D_{1} \neq 0, D_{2}=0$, and $E_{0}=0$. For the examples in this paper we choose $\eta(k)=\bar{\eta}(k) Z^{\mathrm{T}}(k-1) Z(k-1)$, where $\bar{\eta}(k)$ is a nonnegative number.

\section{A. SISO Examples}

Example VIII.1 (SISO MP) Consider the system

$$
A=\left[\begin{array}{ccc}
1.7 & -1.2 & 0.7 \\
1 & 0 & 0 \\
0 & 0.5 & 0
\end{array}\right], B=\left[\begin{array}{l}
2 \\
0 \\
0
\end{array}\right]
$$




$$
D_{1}=\left[\begin{array}{c}
0.9794 \\
-0.2656 \\
-0.5484
\end{array}\right], C=E_{1}=\left[\begin{array}{c}
0.5 \\
-0.65 \\
0.4
\end{array}\right]^{\mathrm{T}},
$$

which is minimum-phase and stable. The goal is to reject the disturbance $w(k)=\sin \left(\frac{\pi}{5} k\right)$. We choose $\tilde{\mathcal{H}}=H_{1}, n_{c}=5, \gamma=1$ and $\bar{\eta}(k)=0$. Figure 2 shows the adaptive controller in closed-loop with the plant. The performance is reduced to zero with knowledge of just one Markov parameter. Furthermore, we are able to choose $\bar{\eta}(k)=0$, since the plant is minimum phase.

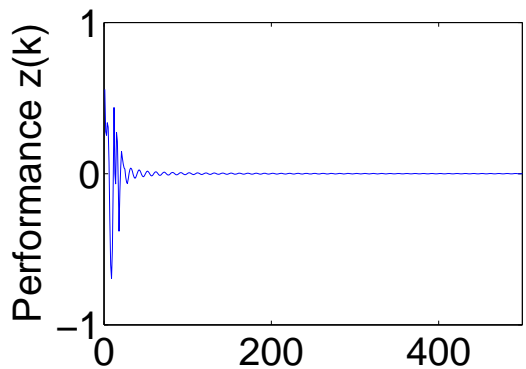

(a)

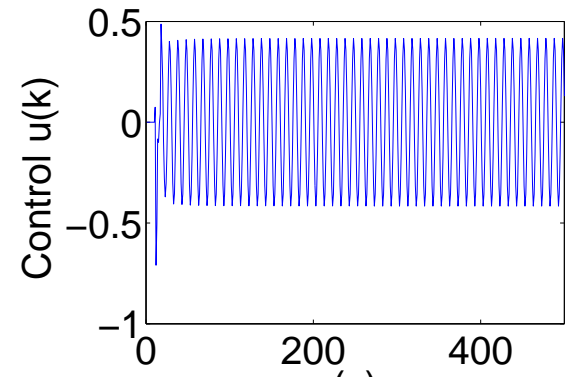

(c)

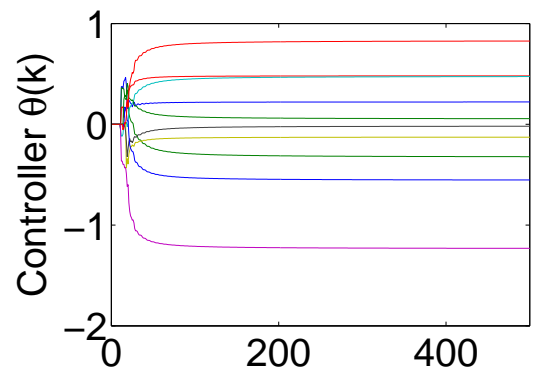

(b)

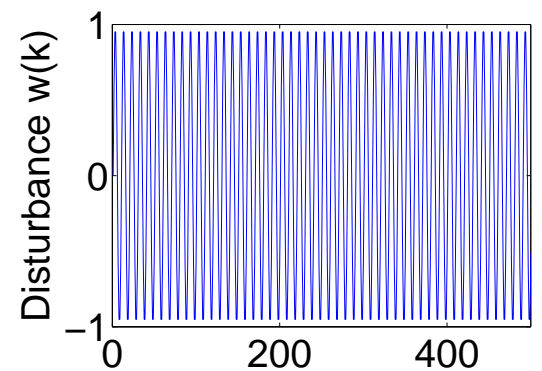

(d)

Figure 2. For this example, the plant is SISO and minimum phase. We choose $\tilde{\mathcal{H}}=H_{1}$ and $\bar{\eta}(k)=0$. (a) shows the performance $z(k)$, (b) shows the controller parameters $\theta(k)$, (c) shows the control signal $u(k)$, and (d) shows the disturbance $w(k)$.

Example VIII.2 (SISO NMP) Consider the system (83) and (84), where $C$ and $E_{1}$ are replaced by

$$
C=E_{1}=\left[\begin{array}{lll}
0.5 & -1.25 & 1
\end{array}\right]
$$

which makes the system nonminimum phase, with a zero at 2 . The goal is to reject the disturbance $w(k)=$ $\sin \left(\frac{\pi}{5} k\right)$. We choose $\tilde{\mathcal{H}}=H_{1}=1, n_{c}=5, \bar{\eta}(k)=2$, and $\gamma=1$. Figure 3 shows the adaptive filter in closed loop with the nonminimum-phase system. Note that the controller does not have any knowledge of the nonminimum-phase zero.

Example VIII.3 (SISO NMP) To examine the effect of $\bar{\eta}(k)$, we reconsider Example 2 with $\bar{\eta}(k)=0$. All other controller parameters are identical. Figure 4 shows the adaptive filter in closed loop with the nonminimum-phase system without adaptive regularization. As the stability analysis predicts, the control signal grows unbounded, which results in unbounded performance as $k$ becomes large.

Example VIII.4 (SISO NMP) Next, we consider the same plant and disturbance as Examples VIII.2 and VIII.3. Furthermore we choose the controller parameters as in VIII.2. However, in this case we assume that we have knowledge of only the $4^{\text {th }}$ Markov parameter, so that $\tilde{\mathcal{H}}=H_{4}=-1.3420$. Figure 5 shows the closedloop performance using knowledge of just the $4^{\text {th }}$ Markov parameter. There is no discernible performance gain or degradation when using $H_{4}$ as opposed to $H_{1}$. 


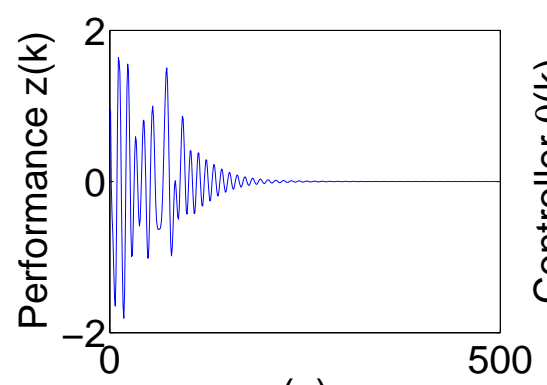

(a)

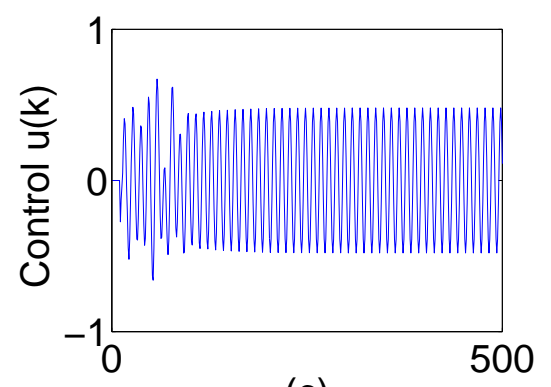

(c)

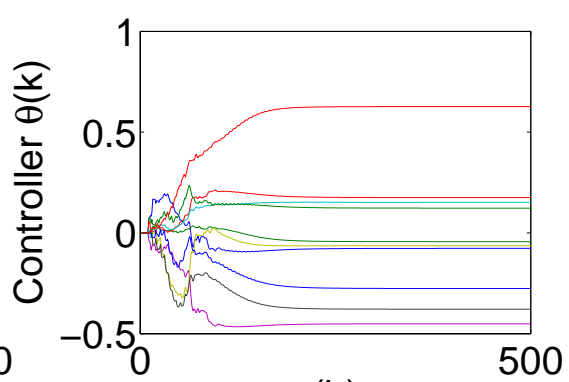

(b)

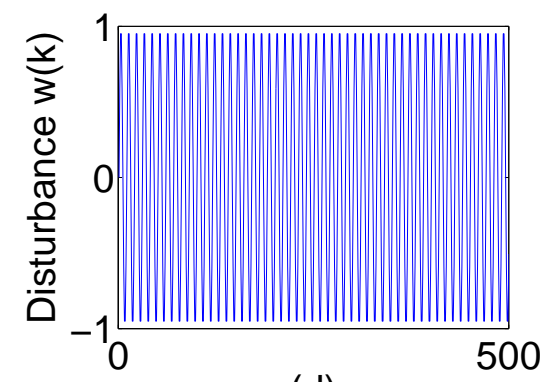

(d)

Figure 3. For this example, the plant is SISO and nonminimum phase. We choose $\tilde{\mathcal{H}}=H_{1}=1$, and $\bar{\eta}(k)=2$. (a) shows the performance $z(k)$, (b) shows the controller parameters $\theta(k)$, (c) shows the control signal $u(k)$, and (d) shows the disturbance $w(k)$.
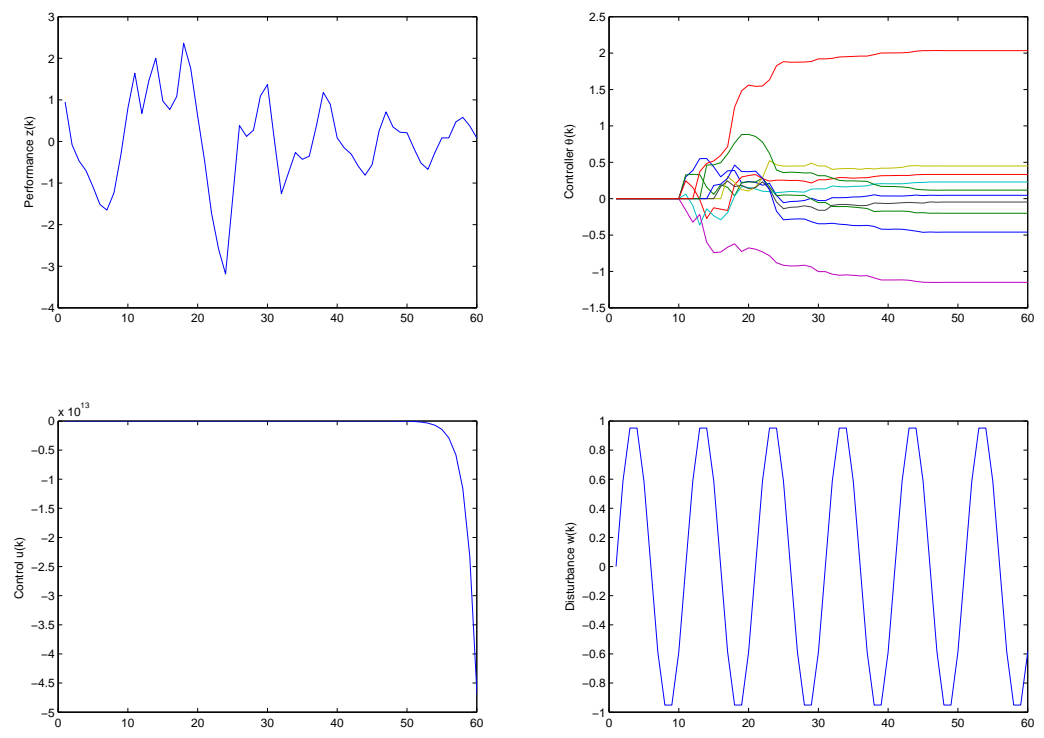

Figure 4. For this example, the plant is SISO and nonminimum phase. We choose $\tilde{\mathcal{H}}=H_{1}$, and $\bar{\eta}(k)=0$. (a) shows the performance $z(k)$, (b) shows the controller parameters $\theta(k)$, (c) shows the control signal $u(k)$, and (d) shows the disturbance $w(k)$. 

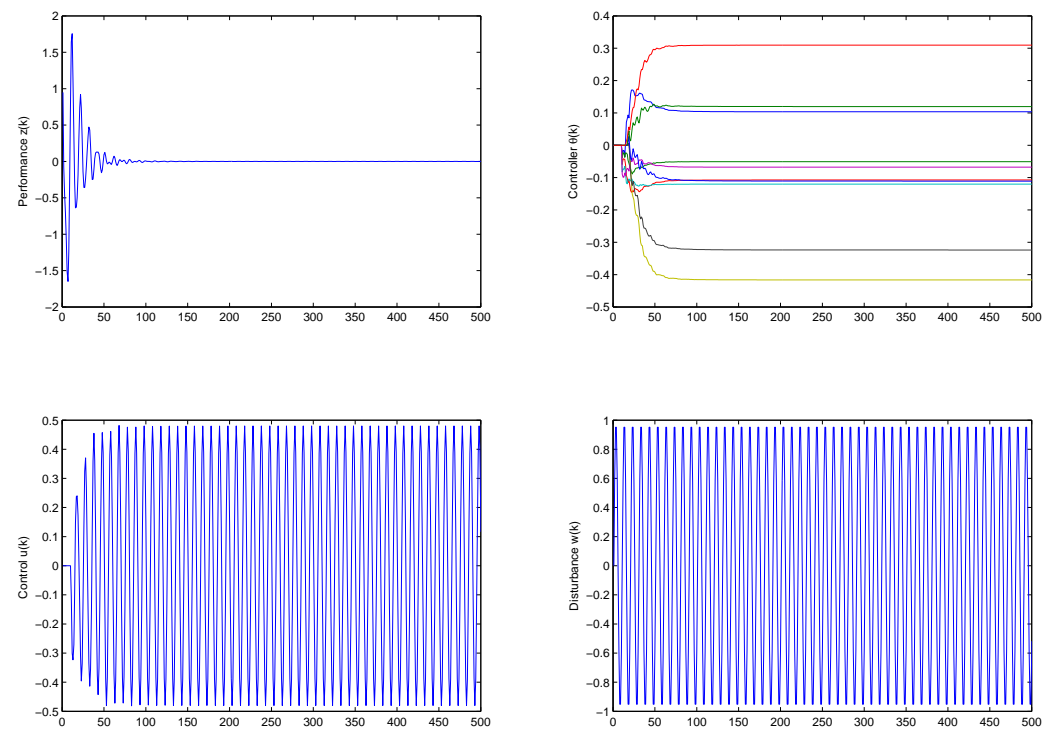

Figure 5. For this example, the plant is SISO and nonminimum phase. We choose $\tilde{\mathcal{H}}=-1.3420$ and $\bar{\eta}(k)=2$. (a) shows the performance $z(k)$, (b) shows the controller parameters $\theta(k)$, (c) shows the control signal $u(k)$, and (d) shows the disturbance $w(k)$.

Example VIII.5 (SISO NMP) We consider the same plant and disturbance as in Example VIII.2. Furthermore we choose the controller parameters as in Example VIII.2. However, in this case assume that the $2^{\text {nd }}$ and $6^{\text {th }}$ Markov parameters are known, and thus $\tilde{\mathcal{H}}=[-0.8-0.1076]^{\mathrm{T}}$. Figure 6 shows the closed-loop performance with knowledge of the $2^{\text {nd }}$ and $6^{\text {th }}$ Markov parameters.

\section{B. MIMO Examples}

Example VIII.6 $(2 \times 2$ NMP) Consider the asymptotically stable system

$$
\begin{gathered}
A=\left[\begin{array}{cccccc}
-0.3 & 0.3 & 0.56 & 0 & 0 & 0 \\
1 & 0 & 0 & 0 & 0 & 0 \\
0 & 0.5 & 0 & 0 & 0 & 0 \\
0 & 0 & 0 & -0.6 & -0.1 & 0.4 \\
0 & 0 & 0 & 1 & 0 & 0 \\
0 & 0 & 0 & 0 & 0.5 & 0
\end{array}\right], \\
B=\left[\begin{array}{ll}
2 & 0 \\
0 & 0 \\
0 & 0 \\
0 & 4 \\
0 & 0 \\
0 & 0
\end{array}\right], D_{1}=\left[\begin{array}{ccc}
0.9794 \\
-0.2656 \\
-0.5484 \\
0.0963 \\
-1.3807 \\
-0.7284
\end{array}\right], \\
C=E_{1}=\left[\begin{array}{cccccc}
0 & 0.5 & 0 & 0.25 & -0.25 & -1 \\
0 & 0 & 1 & 0 & 0.25 & -1
\end{array}\right],
\end{gathered}
$$




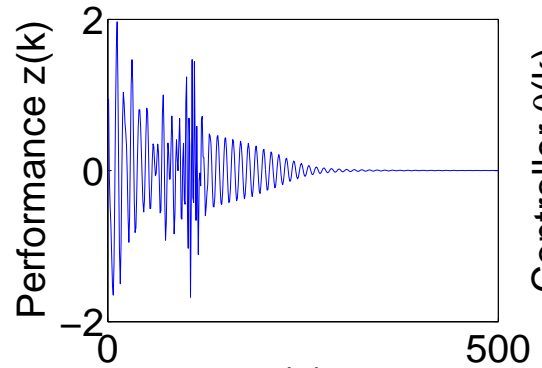

(a)

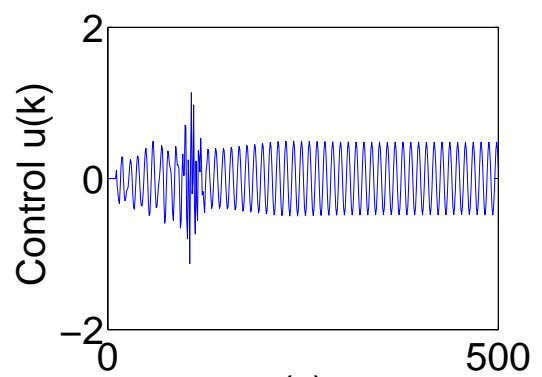

(c)

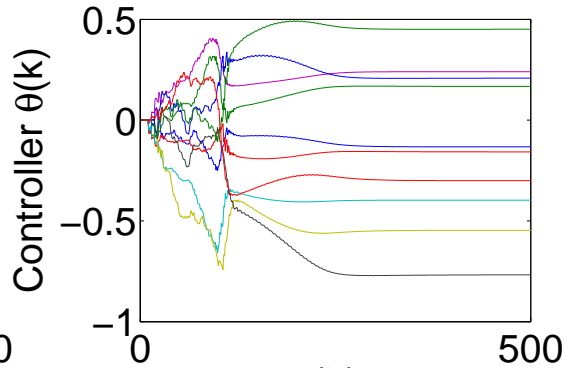

(b)

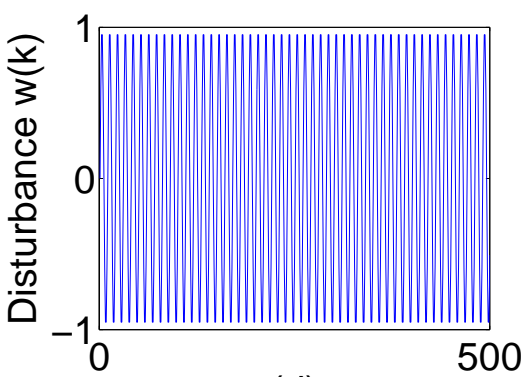

(d)

Figure 6. For this example, the plant is SISO and nonminimum phase. We choose $\tilde{\mathcal{H}}=[-0.8-0.1076]^{\mathrm{T}}$ and $\bar{\eta}(k)=2$. (a) shows the performance $z(k)$, (b) shows the controller parameters $\theta(k)$, (c) shows the control signal $u(k)$, and (d) shows the disturbance $w(k)$.

which has a nonminimum-phase transmission zero at 2 . The goal is to reject the disturbance $w(k)=\sin \left(\frac{\pi}{5} k\right)$. We choose $\tilde{\mathcal{H}}=H_{2}=E_{1} A B=\left[\begin{array}{cc}1 & -1.6 \\ 0 & 1\end{array}\right], n_{c}=5, \bar{\eta}(k)=2$, and $\gamma=1$.

Figure 7 shows closed-loop performance with knowledge of the $2^{\text {nd }}$ Markov parameter. Note in this case that the first nonzero Markov parameter $E_{1} B=\left[\begin{array}{ll}0 & 1 \\ 0 & 0\end{array}\right]$ is not left invertible, and thus another Markov parameter must be used in $\tilde{\mathcal{H}}$, specifically, we used $H_{2}=E_{1} A B$.

Example VIII.7 $(2 \times 1$ NMP) Consider the system

$$
\begin{gathered}
A=\left[\begin{array}{cc}
0.6 & -0.32 \\
0.25 & 0
\end{array}\right], B=\left[\begin{array}{l}
4 \\
0
\end{array}\right], \\
D_{1}=\left[\begin{array}{c}
0.9794 \\
-0.2656
\end{array}\right], C=E_{1}=\left[\begin{array}{cc}
0 & 1 \\
0.25 & -1.5
\end{array}\right],
\end{gathered}
$$

which is minimum phase and asymptotically stable. Note that the channel from the control to the second output has a nonminimum-phase zero at 1.5 . The goal is to reject the disturbance $w(k)=\sin \left(\frac{\pi}{5} k\right)$. We choose $\tilde{\mathcal{H}}=H_{1}=\left[\begin{array}{ll}0 & 1\end{array}\right]^{\mathrm{T}}, n_{c}=5, \gamma=1$, and $\bar{\eta}(k)=1$. Note that $A-B H_{1}^{\dagger} E_{1} A$ has an unstable eigenvalue at 1.5. Figure 8 shows the closed-loop performance with knowledge of the $1^{\text {st }}$ Markov parameter. Note that only one control input is available to reject a disturbance that affects two outputs. It is not possible, in this case, to reduce both performance variables to zero. Consequently, the adaptive controller reduces one performance variable, while the other variable remains at approximately its open-loop value. 


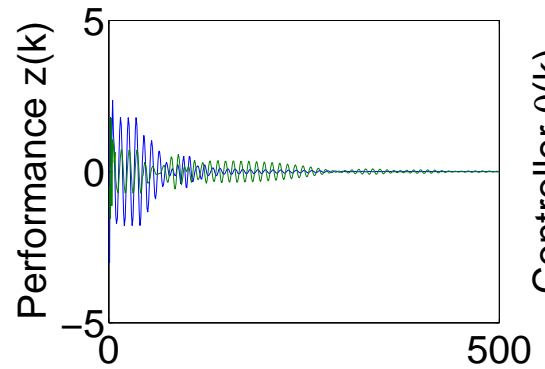

(a)

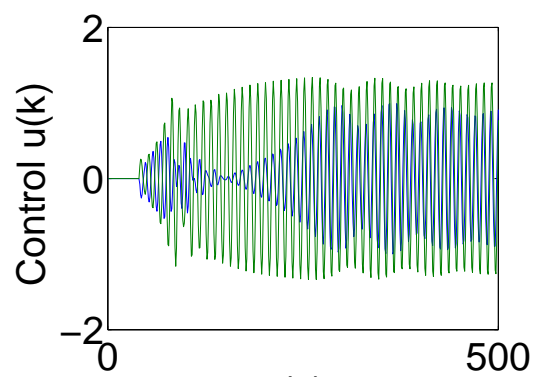

(c)

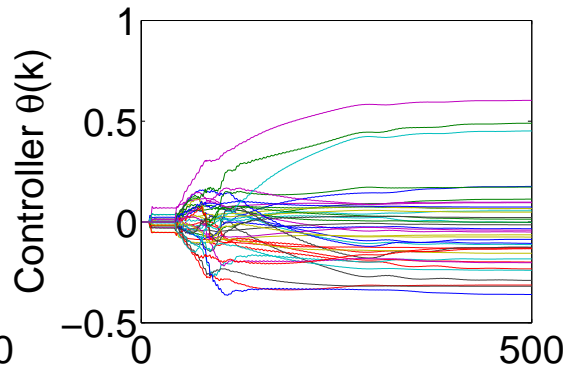

(b)

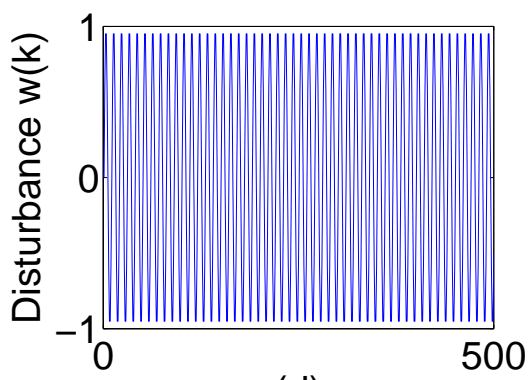

(d)

Figure 7. For this example, the plant is $2 \times 2$ MIMO, and is nonminimum phase. We choose $\tilde{\mathcal{H}}=H_{2}$ and $\bar{\eta}(k)=2$. (a) shows the performance $z(k)$, (b) shows the controller parameters $\theta(k)$, (c) shows the control signal $u(k)$, and (d) shows the disturbance $w(k)$.

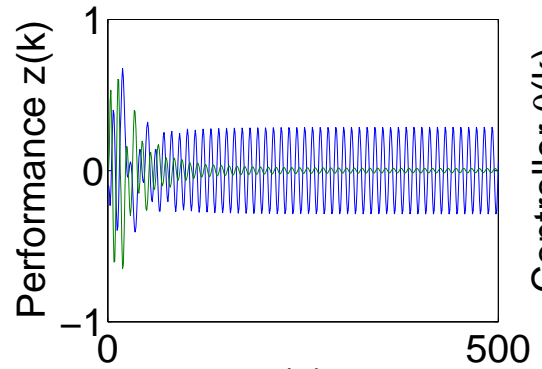

(a)

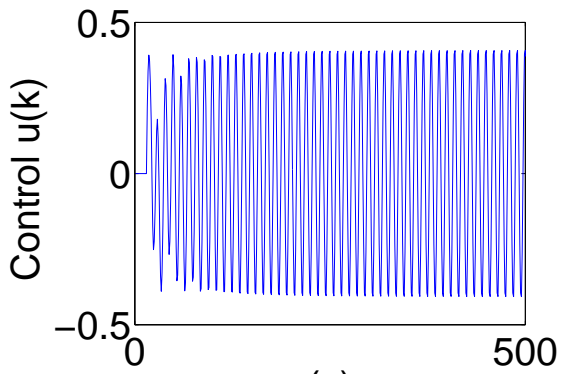

(c)

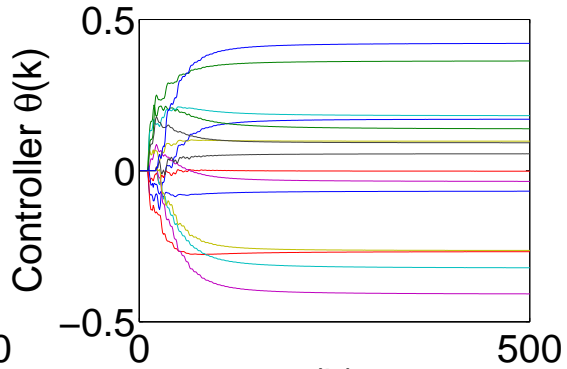

(b)

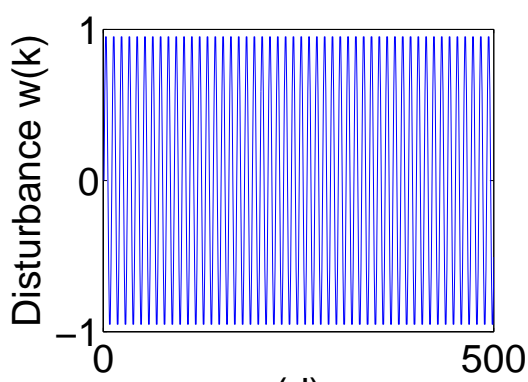

(d)

Figure 8. For this example, the plant is $2 \times 1 \mathrm{MIMO}$, and $A-B H_{1}^{\dagger} E_{1} A$ is unstable. We choose $\tilde{\mathcal{H}}=H_{1}$ and $\bar{\eta}(k)=1$. (a) shows the performance $z(k)$, (b) shows the controller parameters $\theta(k),(\mathbf{c})$ shows the control signal $u(k)$, and (d) shows the disturbance $w(k)$. 
Example VIII.8 $(1 \times 2$ NMP) Consider the asymptotically stable system

$$
\begin{aligned}
A & =\left[\begin{array}{cccc}
0 & 0.4 & 0 & 0 \\
0.5 & 0 & 0 & 0 \\
0 & 00 & -0.1 & 0.4 \\
0 & 0 & 0.5 & 0
\end{array}\right], B=\left[\begin{array}{ll}
2 & 0 \\
0 & 0 \\
0 & 2 \\
0 & 0
\end{array}\right], \\
D_{1} & =\left[\begin{array}{c}
0.9794 \\
-0.2656 \\
-0.5484 \\
0.0963
\end{array}\right], C=E_{1}=\left[\begin{array}{c}
0.5 \\
-1.5 \\
0.5 \\
-1.5
\end{array}\right]^{\mathrm{T}},
\end{aligned}
$$

which has a nonminimum-phase transmission zero at 1.5. The goal is to reject the disturbance $w(k)=$ $\sin \left(\frac{\pi}{5} k\right)$. We choose $\tilde{\mathcal{H}}=\left[\begin{array}{ll}H_{1}^{\mathrm{T}} & H_{2}^{\mathrm{T}}\end{array}\right]^{\mathrm{T}}=\left[\begin{array}{cc}1 & 1 \\ -1.5 & -1.6\end{array}\right], n_{c}=15, \gamma=0.001$, and $\bar{\eta}(k)=0.01$. Figure 8

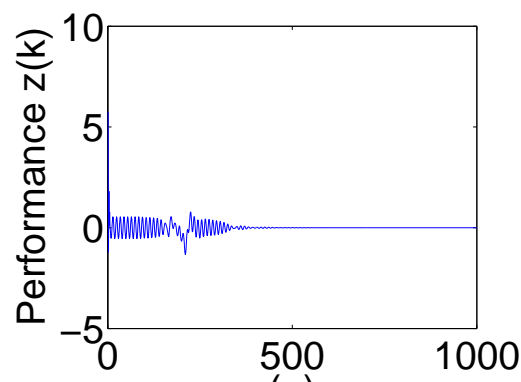

(a)

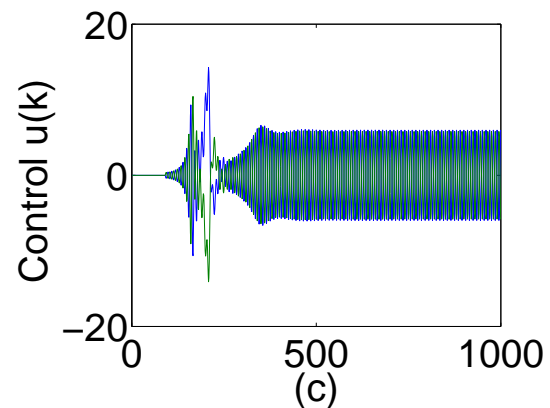

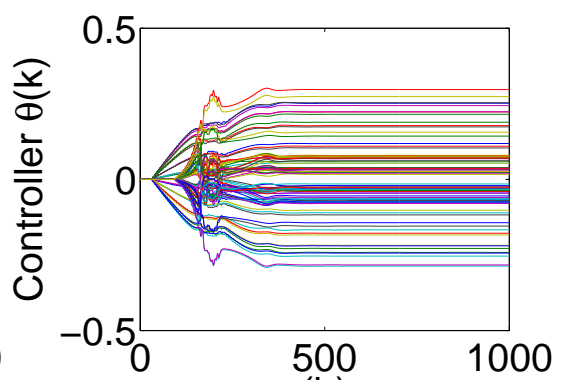

(b)

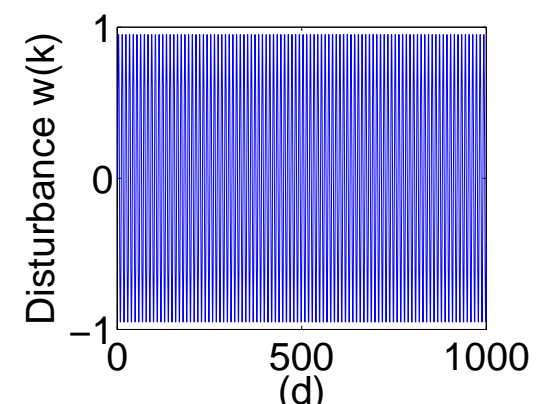

(d)

Figure 9. For this example, the plant is nonminimum phase $1 \times 2$ MIMO. We choose $\tilde{\mathcal{H}}=\left[\begin{array}{ll}H_{1}^{\mathrm{T}} & H_{2}^{\mathrm{T}}\end{array}\right]^{\mathrm{T}}$ and $\bar{\eta}(k)=0.01$. (a) shows the performance $z(k)$, (b) shows the controller parameters $\theta(k)$, (c) shows the control signal $u(k)$, and (d) shows the disturbance $w(k)$.

shows the closed-loop performance with knowledge of the $1^{\text {st }}$ and $2^{\text {nd }}$ Markov parameters. In this case, we note that any single Markov parameter is not left invertible. Therefore, wide systems require more than one Markov parameter to ensure that $\tilde{\mathcal{H}}$ is invertible.

\section{Conclusions}

In this paper we extended the RCAC adaptive control algorithm and investigated its ability to adaptively control systems without knowledge of the nonminimum-phase zeros, if any. Conditions for the stability of the error system were examined in the unregularized and regularized versions of the algorithm. Furthermore, the algorithm was demonstrated on SISO and MIMO examples, starting with a minimum-phase system, and working up to the nonminimum-phase case, including non-square systems. Furthermore, we demonstrated that the algorithm can utilize various combinations of Markov parameters, for example, the $2^{\text {nd }}$ and $6^{\text {th }}$, 
Markov parameters. In all cases, the number of Markov parameters that are used is not sufficient to determine the nonminimum-phase zeros of the system. Consequently, these examples demonstrate the ability to control MIMO nonminimum-phase systems with unknown nonminimum-phase zeros.

\section{References}

${ }^{1}$ K. J. Åström and B. Wittenmark, Adaptive Control, 2 ${ }^{\text {nd }}$ Edition, Addison-Wesley, Reading, MA 1995.

${ }^{2}$ B. D. O. Anderson, "Topical Problems of Adaptive Control", Proc. European Contr. Conf., pp. 4997-4998, Atlanta, GA, July 2007.

${ }^{3}$ E. W. Bai and S. S. Sastry, "Persistency of excitation, sufficient richness and parameter convergence in discrete-time adaptive control", Sys. Contr. Lett. , Vol. 6, pp. 153-163, 1985.

${ }^{4}$ D. S. Bayard, "Stable direct adaptive periodic control using only plant order knowledge", Int. J. Adaptive Contr. Signal Processing, Vol. 10, pp. 551-570, 1996.

${ }^{5}$ R. Venugopal and D. S. Bernstein. "Adaptive Disturbance Rejection Using ARMARKOV System Representations," IEEE Trans. Contr. Sys. Tech., Vol. 8, pp. 257-269, 2000.

${ }^{6}$ J. B. Hoagg, M. A. Santillo, and D. S. Bernstein, "Discrete-Time Adaptive Command Following and Disturbance Rejection for Minimum Phase Systems with Unknown Exogenous Dynamics," IEEE Trans. Autom. Contr., Vol. 53, pp. 912-928, 2008.

${ }^{7}$ M. A. Santillo and D. S. Bernstein, "Adaptive Control Based on Retrospective Cost Optimization," AIAA J. Guid. Contr. Dyn., Vol. 33, pp. 289-304, 2010.

${ }^{8}$ J. B. Hoagg and D. S. Bernstein, "Retrospective Cost Adaptive Control for Nonminimum-Phase Discrete-Time Systems Part 1: The Ideal Controller and Error System, Part 2: The Adaptive Controller and Stability Analysis," Proc. Conf. Dec. Contr., pp. 893-904, Atlanta, GA, December 2010.

${ }^{9}$ J. B. Hoagg and D. S. Bernstein, "Retrospective Cost Model Reference Adaptive Control for Nonminimum-Phase Discrete-Time Systems, Part 2: The Adaptive Controller and Stability Analysis," Proc. Conf. Dec. Contr., pp. 2927-2932, San Francisco, CA, June 2011.

${ }^{10}$ J. B. Hoagg and D. S. Bernstein, "Retrospective Cost Model Reference Adaptive Control for Nonminimum-Phase Discrete-Time Systems, Part 1: The Ideal Controller and Error System," Proc. Conf. Dec. Contr., pp. 2933-2938, San Francisco, CA, June 2011.

${ }^{11}$ E. D. Sumer, A. M. D'Amato, A. M. Morozov, J. B. Hoagg, and D. S. Bernstein, "Robustness of Retrospective Cost Adaptive Control to Markov-Parameter Uncertainty," Proc. Conf. Dec. Contr., Orlando, FL, December 2011. 